\title{
Important role of ventromedial hypothalamic carnitine palmitoyltransferase-1a in the control
}

\section{of food intake}

$\mathrm{Su}_{\text {Gao }}{ }^{1,}$, Dolors Serra ${ }^{2}$, Wendy Keung ${ }^{1}$, Fausto G. Hegardt ${ }^{2}$ and Gary D. Lopaschuk ${ }^{1 *}$

1 From the Department of Pediatrics, Mazankowski Alberta Heart Institute, University of Alberta, Edmonton, AB T6G 2S2, Canada

I Current address: Department of Metabolism and Aging, Scripps Research Institute, Jupiter, FL 33456, USA

${ }^{2}$ Department of Biochemistry and Molecular Biology, Facultat de Farmàcia, Universitat de Barcelona, E-08028 Barcelona, Spain and Institut de Biomedicina de la Universitat de Barcelona (IBUB) and CIBER Fisiopatología de la Obesidad y la Nutrición (CIBERObn), Instituto de Salud Carlos III, Spain.

*To whom correspondence should be addressed:

Dr. Gary D. Lopaschuk, Department of Pediatrics, 423 HMRB

University of Alberta, Edmonton, Alberta T6G 2S2

Canada.

Tel: $780-492-2170$

Fax: 780-492-9753

E-mail: gary.lopaschuk@ualberta.ca

Running title: Activation of VMN CPT-1 increases food intake. 


\section{ABSTRACT}

Carnitine palmitoyltransferase-1 (CPT-1) liver isoform or CPT-1a is implicated in CNS control of food intake. However, the exact brain nucleus site(s) in mediating this action of CPT-1a has not been identified. In this report, we assess the role of CPT-1a in hypothalamic ventromedial nucleus (VMN). We stereotaxically injected an adenoviral vector containing CPT-1a coding sequence into the VMN of rats to induce overexpression and activation of CPT-1a. The VMN-selective activation of CPT-1a induced orexigenic effect, suggesting CPT-1a in the VMN is involved in the central control of feeding. Intracerebroventricular administration of etomoxir, a CPT-1 inhibitor, decreases food intake. Importantly, in the animals with VMN-overexpression of a CPT-1a mutant that antagonizes the CPT-1 inhibition by etomoxir, the anorectic response to etomoxir was attenuated. This suggests that VMN is involved in mediating the anorectic effect of central inhibition of CPT1a. In contrast, Arc overexpression of the mutant did not alter etomoxir-induced inhibition of food intake, suggesting that Arc CPT-1a does not play significant roles in this anorectic action. Furthermore, in the VMN, CPT-1a appears to act downstream of hypothalamic malonyl-CoA action of feeding. Finally, we show that in the VMN, CPT-1 activity altered in concert with fasting and refeeding states, supporting a physiological role of CPT-1a in mediating the control of feeding. Taking together, CPT-1a in the hypothalamic VMN appears to play an important role in the central control of food intake. VMN-selective modulation of CPT-1a activity may therefore be a promising strategy in controlling food intake and maintaining normal body weight. 


\section{INTRODUCTION}

The central nervous system (CNS) regulates energy homeostasis by tightly balancing energy (food) intake, energy expenditure, and energy deposition $(6,47,51)$. The hypothalamus in the CNS plays a crucial role in this process. Diverse neuronal, humoral and nutrient signals indicating energy status converge at the hypothalamus, and the hypothalamus processes and integrates these signals to exert the controls of feeding and energy balance $(18,44,47,48,51)$. It has been shown that fatty acid metabolism plays important roles in the intracellular signaling mechanisms underlying hypothalamic control of energy balance $(12,30,32)$. Carnitine palmitoyltransferase-1 (CPT-1) is a key enzyme in shuttling fatty acid into mitochondria for $\beta$-oxidation, by converting fatty acyl-CoA to acylcarnitine $(36,45)$. Of relevance, it has been proposed that hypothalamic CPT-1 plays a key role in the central control of food intake (39). In the hypothalamus, the predominant CPT-1 isoform possessing acyltransferase activity is the CPT-1 liver isoform (CPT-1a). Previous studies have shown that intracerebroventricular (I.C.V.) administration of CPT-1a selective inhibitors decreases food intake (39). Along with this anorectic effect, I.C.V. delivery of the CPT-1 inhibitors results in the inhibition CPT-1a in the hypothalamic arcuate nucleus (Arc) that is an important site in the control of food intake $(48,51)$. Based on the association of Arc CPT-1a inhibition with the anorectic effects of central CPT-1a inhibitors, it was hypothesized that inhibition of CPT-1a in the Arc contributes to the anorectic effect by central delivery of the inhibitors $(32,39)$. However, since ICV injection targets the entire brain, a specific brain site (such as the Arc) may not necessarily be involved in mediating the global central effect. In line with this argument, our recent studies have demonstrated that modulation of CPT-1a specifically in the Arc fails to alter food intake (15). Instead, in the Arc, the brain isoform of CPT-1 (CPT-1c) that has a rather weak acyltransferase activity $(43,50,58)$, and is not involved in fatty acid oxidation (50), appears to play a role in the control of feeding (17). 
In the peripheral tissues, CPT-1 activity is regulated by the inhibitory action of malonyl-CoA, an intermediate in fatty acid biosynthetic pathway $(36,39)$. Malonyl-CoA in the hypothalamus acts as an anorectic mediator of food intake $(12,30,32)$. The Arc is a site where malonyl-CoA exerts its action on feeding control $(16,17,20,32)$. Since malonyl-CoA inhibits CPT-1 activity, inhibition of CPT-1a in the Arc had been predicted to mediate the downstream effect of Arc malonyl-CoAmediated control of food intake $(32,39)$. However, our recent studies have challenged this prediction, and suggest that Arc malonyl-CoA control of feeding does not act through CPT-1a (but rather through the CPT-1c) (17). Central treatment with leptin or cerulenin (a fatty acid synthase inhibitor) increases malonyl-CoA level in the Arc, which is required for the leptin- or cerulenininduced anorectic action $(15,16,20)$. Unexpectedly, the blockade of malonyl-CoA-mediated inhibition of CPT-1a in the Arc fails to affect the feeding inhibition by either leptin or cerulenin (15). Moreover, our studies have also demonstrated that CPT-1a activity in the Arc does not change under fasting or refeeding condition, although Arc malonyl-CoA levels are altered (15). Taken together, these data have strongly challenged the role of CPT-1a as an intracellular downstream target in Arc malonyl-CoA action on food intake. Our previous studies have further revealed that in the Arc, brain isoform of CPT-1 or CPT-1c may serve as a downstream mediator in malonyl-CoA anorectic signaling action (17).

In addition to the Arc, the mediobasal hypothalamic ventromedial nucleus (VMN) also plays an important role in the central controls of energy balance $(5,7,8,10,13,19,21,24-29,35,41,42,52$, 54-56, 59, 60). As in the Arc, malonyl-CoA metabolism is implicated in VMN-mediated control of feeding (34). Moreover, to our knowledge, there have been no reports on the roles of VMN CPT-1a in the control of food intake. It should also be particularly noted that the original study implicating CPT-1a in the central control of feeding (39) did not address the role of CPT-1a in the VMN (or extra-hypothalamic feeding centers). In this report, we examined the role of CPT1a in the VMN in the control of food intake. We also re-assessed the role of CPT-1a activity in the VMN in the hypothalamic malonyl-CoA control of food intake. 


\section{MATERIALS AND METHODS}

Animal preparations: The animal experiments were performed in accordance with the guidelines and instructions of the Canadian Council for Animal Care, and the experimental procedures were approved by the Animal Policy and Welfare Committee of the University of Alberta. SpragueDawley male rats (225-300 g) were purchased from Charles River Laboratories (MA). The rats were housed in a controlled environment $\left(12 / 12\right.$-h light/dark cycle; $\left.25^{\circ} \mathrm{C}\right)$. They were allowed $\mathrm{ad}$ libitum access to standard laboratory chow and water, unless otherwise noted. Before any experimental treatments, the rats were subjected to daily handling and mock injections in order to minimize the stress from the procedures.

Brain cannulation and intracerebroventricular (I.C.V.) injection: Cannulas were implanted into the third ventricle as described before $(15,16)$. The accuracy of cannula placement was verified by the angiotensin-2 drinking test or by histological analysis (16). After the cannulation surgery, daily body weights were monitored. Once body weights returned to the pre-surgical levels and the rats were fully habituated to the handling and mock injection procedures, the experimental treatments were performed.

Adenoviruses: The recombinant adenoviral vectors having no CPT-1a sequence (null), encoding wild-type CPT-1a, or encoding the mutant CPT-1a were prepared as described previously (15). In brief, the virus encoding wild-type CPT-1a contains the nucleotide sequence covering the coding region of rat CPT-1a (58-2700). This sequence was used to generate the mutant CPT-1a with the methionine residue 593 being mutated to a serine residue (M593S). This M593S mutant CPT-1a is insensitive to malonyl-CoA inhibition, and exhibits reduced response to etomoxir-induced inhibition $(15,37,38)$. The adenoviruses were delivered into the hypothalamic VMN or Arc by bilateral stereotaxic injection $\left(1 \times 10^{7}\right.$ p.f.u. $/ \mu \mathrm{l} ; 0.4 \mu \mathrm{l}$ per side $)$. The coordinates of VMN injection are: anterior-posterior: $-2.8 \mathrm{~mm}$; dorsal-ventral: $-9.1 \mathrm{~mm}$; and medial-lateral: $\pm 0.6-0.7 \mathrm{~mm}$. The accuracy of the injections was tested elsewhere (addressed in a separate manuscript). The coordinates of Arc injection are: anterior-posterior: $-2.8 \mathrm{~mm}$; dorsal-ventral: $-9.5 \mathrm{~mm}$; and medial- 
lateral: $\pm 0.4 \mathrm{~mm}$. The accuracy of the Arc injection has been verified and documented in our previous report (15). Significantly high levels of CPT-1a protein or enzyme activity were detected starting at around 7 days following the viral injections, and the expression levels lasted until at least 14 days following the viral injections.

Chemicals: Etomoxir was obtained either from Sigma (MO) or as a generous gift of Dr. Grant M. Hatch at the University of Manitoba. Etomoxir was dissolved in phosphate buffered saline (PBS), and a dose of $108 \mu \mathrm{g}$ (320 nmole) was administered by I.C.V. bolus injection, at $2 \mathrm{~h}$ before the onset of the dark cycle (when food is presented). This dose was chosen since a study reported that I.C.V. treatment of mice with etomoxir at this level robustly reduces body weight (1). Cerulenin (Sigma, MO) was dissolved in the vehicle of $25 \%$ DMSO in PBS, and a dose of $125 \mu \mathrm{g}$ was administered by I.C.V. bolus injection (15). The cerulenin injection was also performed before onset of the dark cycle.

Brain sample preparations: At the designated time points, the rats were euthanized by decapitation. The brains were dissected within 1 minute, and coronal hypothalamic sections were prepared using a cryostat or a brain matrix (Roboz Surgical Instrument, Gaithersburg, MD). The arcuate nucleus (Arc) and the ventromedial nucleus (VMN) were dissected based on the established protocol (15, 16).

Quantifications of hypothalamic malonyl-CoA, long-chain acyl-CoA's, and long-chain acylcarnitines: Malonyl-CoA levels were quantified by the CoA recycling assay (16). The levels of long-chain acyl-CoA's (consisting of palmitoyl-CoA, oleoyl-CoA, and stearoyl-CoA) and acylcarnitines (consisting of palmitoylcarnitine, oleoylcarnitine, and stearoylcarnitine) were measured by HPLC and mass spectrometry (15).

Carnitine palmitoyltransferase-1 (CPT-1) activity assay: The brain was removed within 40 seconds following decapitation, and was immediately sectioned using the brain matrix. The VMN areas were quickly dissected, and subjected to the CPT-1 activity assay, as described previously (15). 
Antibodies and Western blotting: The anti-CPT-1a and CPT-1c antibodies were described previously (15). Anti- $\alpha$-tubulin antibody (Cell Signaling) was used to detect $\alpha$-tubulin as the loading control. The procedures of electrophoresis, transfer, and signal detection were based on the Western blotting analysis protocol (Invitrogen). Protein band was quantitated by densitometry using Scion Image software (Scion).

Statistical analysis: Data are presented as the mean \pm standard error of the mean (S.E.M.). Data consisting of two groups were analyzed using the Student t-test. Data consisting of multiple groups were analyzed by one-way or two-way ANOVA. These ANOVAs, when they yielded significant overall effects, were further analyzed by the Newman-Keuls test or Bonferroni post test for group comparisons. For all tests, $\mathrm{P}<0.05$ was considered significant.

\section{RESULTS}

Activation of CPT-1a induces orexigenic effects: In the hypothalamus, the predominant CPT-1 isoform possessing acyltransferase activity is the liver-isoform (CPT-1a) (39). To explore the VMN-specific role of CPT-1a in the control of feeding, we injected the recombinant adenoviral vector encoding wild type CPT-1a (CPT-1a wt) or the malonyl-CoA insensitive mutant CPT-1a (CPT-1a mt) into the VMN of rats. The adenoviral vector without the CPT-1a insert (CPT-1a null) was used as the control. After one week following the viral injections the rats were euthanized and the CPT-1 protein levels in the VMN area were measured by Western blotting. Compared with the rats injected with the null virus, CPT-1a (either wt or mt) viral infections induced increases in CPT1a protein levels in the VMN (Fig. 1). In addition to CPT-1a, the brain isoform of CPT-1 or CPT$1 \mathrm{c}$ is also expressed in hypothalamic nuclei $(43,58)$. Following the viral injection, there were no detectable differences of CPT-1c protein levels in the VMN among the groups (Fig. 1), which confirms the isoform-specific overexpression following the viral infection. 
The upregulations of the protein levels of CPT-1a wt and CPT-1a mt were associated with significant increases in the CPT-1 activities in both CPT-1a wt and CPT-1a mt groups (Fig. 2A). Confirming the specificity of VMN expression, we observed no changes in CPT-1 activity in the other hypothalamic sites, including the PVN and the LHA (Fig. 2A). In the current study, we did not measure CPT-1 activity in the Arc area because we could not obtain sufficient Arc tissue lysates following dissecting VMN from the same animal. However, we did measure the levels of longchain acylcarnitines (LC-AC's) in these two nuclei (VMN and Arc). LC-AC's are direct products of CPT-1a activity, and therefore the measurement of LC-AC's levels has been used as a surrogate assay of CPT-1a activity $(15,23)$. The typical acyltransferase activity of CPT-1 converts palmitoyl-, oleoyl- and stearoyl-CoA, the major LCFA-CoA species, to palmitoyl-, oleoyl- and stearoylcarnitine, the major LC-AC species, respectively $(15,23)$. We quantified the levels of these LCAC's in the VMN and the Arc regions. Their levels in the CPT-1a (wt and mt) animals were increased in the VMN, but were not altered in the Arc (Fig. 2B), which demonstrates the VMNselective activation of CPT-1a within the mediobasal hypothalamus encompassing the VMN and the Arc. .

In our previous study in which the CPT-1a wt and CPT-1a mt adenoviruses were injected into the Arc, we did not detect any significant changes in food intake or body weight in the CPT-1a (wt and $\mathrm{mt}$ ) groups, as compared to the null control (15). To determine whether VMN overexpression of CPT-1a wt or CPT-1a mt would alter feeding behavior, we injected the same amount of the viruses into the VMN that we previously used in the Arc study, and then monitored daily food intake and body weight. In contrast with the Arc findings, VMN overexpression of CPT-1a (wt and $\mathrm{mt}$ ) induced increases in both food intakes starting on the 5th day (day 5) following viral injections (Fig. 3A), and body weight gains starting on the 4th day (day 4) following viral injections (Fig. 3B). These increases lasted at least until the 8th day (day 8) following the viral injections (Fig. 3A and B). Thus, our data demonstrate that VMN-selective activation of CPT-1a can induce orexigenic 
effects. It should be noted that the CPT-1 mt induced a greater increase in food intake than the CPT1a wt on the 8th day following viral injection. Since the mutant CPT-1a is insensitive to malonylCoA inhibition, overexpression of this mutant should result in a greater in vivo increase in CPT-1a activity (than the overexpression of the wild type), due to a greater resistance to endogenous malonyl-CoA inhibition. This would account for the observed greater orexigenic response in the mutant group. Alteration of CPT-1a activity can affect the levels of long-chain acyl-CoA's (LCFACoA's) that are the substrates of CPT-1a, and the LCFA-CoA's were believed to act as downstream effectors in mediating hypothalamic CPT-1a action on feeding $(39,40)$. We therefore measured the LCFA-CoA's levels in the VMN. Around the time period when the robust CPT-1 activations were detected (i.e., after 7 days following the viral injections), the LCFA-CoA's levels were found to decrease in the VMN (Fig. 3C). This finding further confirms the activation of CPT-1a in the VMN following the viral infection.

Activation of CPT-1a in the VMN blocks central etomoxir-induced anorectic effects: Previous studies examining the role of CPT-1a in feeding behavior did not identify the target brain site in mediating the anorectic effect by I.C.V. administration of CPT-1a inhibitors (39). Having shown that CPT-1a in the VMN plays a role in feeding control, we explored the potential involvement of VMN in mediating the anorectic effects exerted by central CPT-1a inhibition. To this end, we used etomoxir that is a well-known inhibitor of CPT-1 activity (9). Inside the cell, etomoxir is converted to etomoxiryl-CoA, and etomoxiryl-CoA binds CPT-1 and inhibits its activity, partially mimicking the inhibitory action of malonyl-CoA on CPT-1 activity (37). Importantly, it has been demonstrated that the malonyl-CoA insensitive mutant CPT-1a is also resistant to etomoxiryl-CoA-mediated inhibition (37). We centrally (by I.C.V.) injected etomoxir to the rats with VMN-overexpression of either CPT-1a wt or CPT-1a mt. Etomoxir treatment reduced LC-AC's levels (Fig. 4A), and elevated LCFA-CoA's levels (Fig. 4B) in the VMN in both null and CPT-1a wt rats, which demonstrates the inhibitory action of etomoxir on CPT-1a activity in the VMN. Overexpression of 
CPT-1a mt partially blocked these changes (Fig. 4A and 4B), indicating the inhibitory effect of etomoxir treatment on CPT-1a activity was attenuated. Next, we monitored food intake and body weigh change in these animals. We showed that central etomoxir reduced food intakes and induced weight losses in the null and CPT-1a wt animals (Fig. 4C and 4D). These results are consistent with the previous findings from different laboratories that demonstrate the anorectic effects induced by I.C.V. CPT-1 inhibitors $(37,39)$. Importantly, we found that VMN-overexpression of the CPT-1a $\mathrm{mt}$ attenuated the anorectic and weight loss effects of central etomoxir administration (Fig. 4C and 4D). These results show that CPT-1a in the VMN is involved in mediating the feeding response by I.C.V. etomoxir.

\section{Activation of CPT-1a in the Arc does not block central etomoxir-induced anorectic effects:}

The original report addressing brain CPT-1a feeding effect demonstrates an association of the inhibition of CPT-1a in the Arc with anorectic response following I.C.V. delivery of CPT-1a inhibitors (39). To assess the potential causal role of the Arc in mediating the feeding response, we injected (by I.C.V.) etomoxir into the rats with Arc-overexpression of CPT-1a. The Arc-selective overexpression and the activation of CPT-1a activity have been demonstrated in our previous study (15). In the current study we show that I.C.V. etomoxir decreased LC-AC's and increased LCFACoA's levels in the Arc in null and CPT-1a wt rats (Fig. 5A and 5B). These changes confirm the inhibitory effect of central etomoxir treatment on CPT-1a activity in the Arc. As in the VMN, Arcoverexpression of the CPT-1a mt blocked the etomoxir-induced inhibition of CPT-1a activity (Fig. 5A and 5B). However, unlike in the VMN, we found that central etomoxir reduced food intakes and body weights to the same extents across the three groups (i.e. null, CPT-1a wt and CPT-1a mt) (Fig. 5C and 5D). These data demonstrate that the blockade of CPT-1a inhibition (by etomoxir) in the Arc does not affect the anorectic effects following central etomoxir.

Activation of CPT-1a in the VMN blocks malonyl-CoA-mediated anorectic effects: As in the 
Arc, malonyl-CoA in the VMN acts as anorectic mediator in hypothalamic control of feeding (34). In addition, fasting and refeeding states induce VMN-specific changes in the expression levels of fatty acid synthase (FAS) that uses malonyl-CoA as the substrate (34). We then assessed whether the inhibition of CPT-1a activity would act downstream of VMN malonyl-CoA-mediated feeding effect. To this end, we centrally treated (by I.C.V.) animals with cerulenin, a potent FAS inhibitor $(31,53)$, in order to induce an increase in malonyl-CoA levels in the VMN. It has been shown that FAS inhibition in the VMN induces anorectic response, and the increase in malonyl-CoA level that is required for the feeding inhibition (34). We first confirmed that I.C.V. cerulenin increased malonyl-CoA levels in the VMN in the null rats (\% vehicle; vehicle-treated: $100 \pm 2.5$, cerulenintreated: $168 \pm 15 ; \mathrm{P}<0.05)$. We then injected cerulenin to the rats overexpressing CPT-1a in the VMN. Cerulenin treatment produced anorectic effects and weight losses in the rats overexpressing null or CPT-1a wt (Fig. 6A and 6B). Importantly, VMN overexpression of the CPT-1a mt (which is insensitive to malonyl-CoA inhibition) attenuated these changes (Fig. 6A and 6B). Since the feeding action of central cerulenin is attenuated when malonyl-CoA inhibition of CPT-1a is antagonized, the inhibition of CPT-1a is a significant step in the malonyl-CoA signaling action in cerulenin anorectic feeding effect (in the VMN).

\section{Malonyl-CoA levels and CPT-1a activity in the VMN alter in concert under fasting and}

refeeding states: Our data suggest that alterations in CPT-1a activity are involved in the VMNmediated effect on feeding. We further addressed the physiological relevance of these changes by examining the activity of the CPT-1a in the VMN under fasting and re-feeding conditions. Fasting, a negative energy balance condition, reduced malonyl-CoA level in the VMN, while refeeding, a positive energy balance condition, increased malonyl-CoA to the fed level (Fig. 7A). These malonyl-CoA level changes are likely driven by the alterations of the activities of AMPK and ACC. Fasting increases the phosphorylation level of AMPK indicating activation and the phosphorylation level of ACC indicating inhibition, while refeeding reverses these changes (unpublished data). We 
also measured VMN levels of long-chain acyl-CoA's (the substrates of CPT-1a) and acylcarnitines (the products of CPT-1a), as a measure of endogenous CPT-1a activity. The levels of long-chain acylcarnitines increased in the fasting state, while decreased in the refeeding state (Fig. 7B). Furthermore, we show that fasting condition reduced long-chain acyl-CoA's levels, while refeeding condition elevated them (Fig. 7C). Taken together, our data show that CPT-1a activity increases in the fasting state when malonyl-CoA level decreases, while CPT-1a activity decreases in the refeeding state when malonyl-CoA level increases. Thus, in the VMN, CPT-1a activities are altered in concert with malonyl-CoA levels under fasting and refeeding conditions.

\section{DISCUSSION}

The current studies show that modulation of CPT-1a activity in the hypothalamic ventromedial nucleus is sufficient to alter food intake. The data suggest that the VMN is a key site in mediating the actions of central CPT-1a in the control of feeding.

Our study reproduced the finding (37) that I.C.V. administration of etomoxir induces anorectic actions. This result is also in line with the previous observation that central administration of specific CPT-1a inhibitors (CPT-1a-ribozyme and compound ST-1326) reduces food intake (39). However, these data seem to be inconsistent with the finding that central treatment with compound C89b, a putative CPT-1 activator, inhibits food intake (1). Central administration of C89b had been expected to increase food intake rather than inhibit feeding. In this study, it was concluded that C89b acts as a CPT-1 activator, based entirely on an in vitro assay (1). However, the actual effects of C89b treatment on brain CPT-1 acyltransferase activity have not been tested, and therefore the in vivo action of $\mathrm{C} 89 \mathrm{~b}$ on $\mathrm{CPT}-1$ has remained primarily presumptive. As a result, the validity of using compound $\mathrm{C} 89 \mathrm{~b}$ as an "activator" of CPT-1 in in vivo studies is questionable. In contrast, the molecular actions of either etomoxir or CPT-1a-ribozyme (CPT-1a-Ribo) and ST-1236 on brain CPT-1a activity have been fully verified using in vivo approaches. Furthermore, in the C89b study, the specificity of C89b-mediated action has not been assessed, and the potential non-specific effects 
may have played a predominant role in altering food intake. In our etomoxir study, however, we believe the action on CPT-1a plays a significant role in mediating the observed feeding effects. The overexpression of the mutant CPT-1a (insensitive to the etomoxiryl-CoA-mediated inhibition) would not block the etomoxir-induced feeding action, if etomoxir action on CPT-1a did not play a significant role in the observed change in food intake.

The downstream mediators of hypothalamic CPT-1a action on feeding are not clear. It has been reported that I.C.V. infusion of oleic acid (a major long-chain fatty acid) reduces food intake (40, 49). Because fatty acids can be converted into acyl-CoA, oleic acid-derived oleoyl-CoA may mediate the observed anorectic effect (40). Furthermore, since they are the substrates of CPT-1a, LCFA-CoA's such as oleoyl-CoA were proposed to mediate the downstream effect of hypothalamic CPT-1 action on feeding (39). However, some other data do not fully support these hypothesized roles of LCFA-CoA's (15). LCFA-CoA's consist of CoA species with different lengths and saturation degrees, rendering them with distinct properties and functions (46). As a result, the actions of oleoyl-CoA can be different from those of the other LCFA-CoA's. Indeed, unlike central oleic acid, I.C.V. infusion of palmitic acid (another major long-chain fatty acid), which can increase hypothalamic palmitoyl-CoA level, did not affect feeding (49). We have also shown that intrahypothalamic administration of palmitic acid acutely increases food intake (personal communication with Dr. Timothy H. Moran, Johns Hopkins University School of Medicine, Baltimore, MD). Consistent with this, high-fat saturated (palmitic acid) diets attenuate the hypothalamic anorectic signaling actions of leptin and insulin (4). Thus, individual LCFA-CoA's exhibit differential actions on the control of feeding, and LCFA-CoA does not possess one single generic effect on feeding.

In peripheral tissues such as liver, CPT-1 is a rate-limiting enzyme in mitochondrial fatty acid oxidation (FAO) pathway (57). In addition to affecting substrate levels, modulation of CPT-1 activity can impact fatty acid oxidation (FAO) and the production of FAO-derived mitochondrial reactive oxygen species (ROS). In turn, ROS levels can affect the activity and/or the level of 
uncoupling proteins (UCP) that play a role in regulating ROS homeostasis (3). Of relevance, it has been shown that hypothalamic UCP-2 is implicated in ghrelin's hypothalamic action on feeding (2). Ghrelin treatment activates CPT-1 activity in the hypothalamus (33), which leads to upregulation of hypothalamic UCP-2 message level (2). The increased action of UCP-2 limits the elevated production of ROS level resulting from the activation of CPT-1-mediated FAO (by ghrelin) (2). As increase in hypothalamic ROS level can induce anorectic effects $(11,14,22)$, UCP-2 seems to play a permissive role in ghrelin's orexigenic effect. Indeed, we found that overexpression of CPT-1a (in the VMN) induces a significant upregulation of UCP-2 message level, which may restrict the potential overproduction of ROS associated with CPT-1 activation (addressed in another manuscript). Thus, UCP-2 is implicated, and might serve as a mediator, in the downstream signaling pathways of CPT-1a action on feeding control.

A previous study (39) demonstrated an association of the reduction of Arc CPT-1a activity with the anorectic effect, following I.C.V. treatment with CPT-1a-Ribo or ST-1326 (specific CPT-1a inhibitors). Primarily based on this association, the inhibition of CPT-1a in the Arc was predicted to account for the observed anorectic actions (39). Since I.C.V. administration target many brain sites, and association does not indicate causality, the CPT-1a in the Arc may not necessarily mediate the feeding response. Indeed, our current data show that the CPT-1a in the VMN plays an important role in mediating etomoxir-induced anorectic effect. For comparison with the original study (39), we also investigated the role of Arc CPT-1a in etomoxir-induced feeding inhibition. Following I.C.V. etomoxir, the CPT-1a activity in the Arc was reduced to a similar degree in the Arc as it was in the VMN. However, unlike in the VMN, blockade of etomoxir inhibition of CPT-1a in the Arc does not affect the anorectic effect of etomoxir. Thus, using etomoxir as a model compound for inhibiting CPT-1 acyltransferase activity, our studies suggest that the VMN, but not the Arc, is a site in mediating the anorectic effect of central CPT-1a inhibition. To further support a site-specific effect of CPT-1a, VMN-selective activation of CPT-1a activity was found to stimulate feeding (Fig. 3A and 3B), while the Arc-selective activation of CPT-1a (to a similar degree as in the VMN) was 
shown not to alter food intake (15). The differential actions of CPT-1a between the VMN and the Arc are also evident under physiological conditions. The fasting-refeeding data demonstrates that the CPT-1a activity in the VMN is regulated by energy balance conditions, supporting the physiological relevance of the activity change. In contrast, our previous report shows that CPT-1a activity in the Arc is not altered by the fasting-refeeding cycle (15). Taken together, these data have failed to support the significant role of Arc CPT-1a in the central control of feeding as was originally proposed. Instead, the CPT-1a in the VMN appears to play more important roles in the control of food intake. The underlying mechanisms of the differential actions of CPT-1a in the two nuclei are currently unknown. Identification of the downstream mediators of CPT-1a feeding action will be necessary to tackle this question. .

The differential roles of CPT-1a is also implicated in the hypothalamic malonyl-CoA feeding mechanism. The malonyl-CoA-mediated control of food intake is operative in both the Arc and the VMN $(32,34)$. Our previous studies demonstrate that Arc overexpression of the malonyl-CoA insensitive (mutant) CPT-1a does not affect the anorectic response following I.C.V. cerulenin (15). This indicates that in the Arc, malonyl-CoA inhibition of CPT-1a is not an important step in the cerulenin-induced anorectic effect that requires the increase in malonyl-CoA level (15). Furthermore, although the increase in Arc malonyl-CoA level is necessary in leptin's anorectic intracellular signaling pathways $(15,16,20)$, the malonyl-CoA-mediated inhibition of CPT-1a is also not required in leptin's anorectic action (15). Taken together, these studies show that in the Arc, malonyl-CoA acts independent of the inhibition of CPT-1a activity in the control of food intake (15). The current studies demonstrate that in the VMN, malonyl-CoA-mediated inhibition of CPT-1a activity is required for the feeding inhibition of central cerulenin treatment. Thus, unlike in the Arc, in the VMN, CPT-1a may act as an intracellular downstream target in malonyl-CoAmediated effect on feeding. The site-specific roles of CPT-1a in malonyl-CoA feeding mechanisms are also relevant under physiological conditions. Our previous study demonstrates that in the Arc, CPT-1a activities do not alter, although malonyl-CoA levels change in response to fasting and 
refeeding cycle (15). In contrast, the current data show that the CPT-1a activities in the VMN are altered in concert with changes in malonyl-CoA levels, under fasting and refeeding. As an anorectic mediator, hypothalamic malonyl-CoA level decreases during fasting, which would promote feeding. Following refeeding, malonyl-CoA level increases, which would constrain the rebound feeding. Thus, in the VMN, malonyl-CoA likely targets CPT-1a to control feeding and maintain normal energy balance. Concerning the underlying mechanisms of the differential actions, neuronal heterogeneity might play a role. In the Arc, the major enzymes for malonyl-CoA metabolism such as FAS might not be present in the cells that express CPT-1a. On the other hand, they may be coexpressed in the VMN.

In summary, we have identified the VMN as a site in mediating the action of central CPT-1a activity in feeding control. In contrast, our data argue against a significant role of Arc CPT-1a in the hypothalamic control of food intake. Furthermore, in the VMN, but not in the Arc, CPT-1a appears to act downstream of malonyl-CoA effect on food intake. Thus, VMN-selective modulation of CPT-1 acyltransferase activity would provide a strategy to control food intake and regulate body weight.

\section{ACKNOWLEDGEMENTS}

These studies were funded by a grant from the Canadian Diabetes Association and a fellowship from Heart and Stroke Foundation of Canada (awarded to S. Gao). We thank Amy Barr (University of Alberta, Alberta, Canada) for the preparation (amplification) of the recombinant adenoviruses. We also thank Ken Strynadka and Thomas Panakkezhum (University of Alberta, Alberta, Canada) for the HPLC analysis of acyl-CoA. 


\section{REFERENCES}

1. Aja S, Landree LE, Kleman AM, Medghalchi SM, Vadlamudi A, McFadden JM, Aplasca A, Hyun J, Plummer E, Daniels K, Kemm M, Townsend CA, Thupari JN, Kuhajda FP, Moran TH, and Ronnett GV. Pharmacological stimulation of brain carnitine palmitoyl-transferase-1 decreases food intake and body weight. American journal of physiology 294: R352-361, 2008.

2. Andrews ZB, Liu ZW, Wallingford N, Erion DM, Borok E, Friedman JM, Tschop MH, Shanabrough M, Cline G, Shulman GI, Coppola A, Gao XB, Horvath TL, and Diano S. UCP2 mediates ghrelin's action on NPY/AgRP neurons by lowering free radicals. Nature 454: 846-851, 2008.

3. Arsenijevic D, Onuma H, Pecqueur C, Raimbault S, Manning BS, Miroux B, Couplan E, Alves-Guerra MC, Goubern M, Surwit R, Bouillaud F, Richard D, Collins S, and Ricquier D. Disruption of the uncoupling protein-2 gene in mice reveals a role in immunity and reactive oxygen species production. Nature genetics 26: 435-439, 2000.

4. Benoit SC, Kemp CJ, Elias CF, Abplanalp W, Herman JP, Migrenne S, Lefevre AL, Cruciani-Guglielmacci C, Magnan C, Yu F, Niswender K, Irani BG, Holland WL, and Clegg DJ. Palmitic acid mediates hypothalamic insulin resistance by altering PKC-theta subcellular localization in rodents. The Journal of clinical investigation 119: 2577-2589, 2009.

5. Butera PC. Estradiol and the control of food intake. Physiology \& behavior 99: 175$180,2010$.

6. Butler AA and Kozak LP. A recurring problem with the analysis of energy expenditure in genetic models expressing lean and obese phenotypes. Diabetes 59: 323-329, 2010.

7. Chao H, Digruccio M, Chen P, and Li C. Type 2 corticotropin-releasing factor receptor in the ventromedial nucleus of hypothalamus is critical in regulating feeding and lipid metabolism in white adipose tissue. Endocrinology 153: 166-176, 2012.

8. Chen P, Vaughan J, Donaldson C, Vale W, and Li C. Injection of Urocortin 3 into the ventromedial hypothalamus modulates feeding, blood glucose levels, and hypothalamic POMC gene expression but not the HPA axis. American journal of physiology Endocrinology and metabolism 298: E337-345, 2010.

9. Declercq PE, Falck JR, Kuwajima M, Tyminski H, Foster DW, and McGarry JD. Characterization of the mitochondrial carnitine palmitoyltransferase enzyme system. I. Use of inhibitors. The Journal of biological chemistry 262: 9812-9821, 1987.

10. Dhillon H, Zigman JM, Ye C, Lee CE, McGovern RA, Tang V, Kenny CD, Christiansen LM, White RD, Edelstein EA, Coppari R, Balthasar N, Cowley MA, Chua S, Jr., EImquist JK, and Lowell BB. Leptin directly activates SF1 neurons in the VMH, and this action by leptin is required for normal body-weight homeostasis. Neuron 49: 191-203, 2006.

11. Diano S, Liu ZW, Jeong JK, Dietrich MO, Ruan HB, Kim E, Suyama S, Kelly K, Gyengesi E, Arbiser JL, Belsham DD, Sarruf DA, Schwartz MW, Bennett AM, Shanabrough M, Mobbs CV, Yang X, Gao XB, and Horvath TL. Peroxisome proliferation-associated control of reactive oxygen species sets melanocortin tone and feeding in diet-induced obesity. Nature medicine 17: 1121-1127, 2011.

12. Dowell $\mathbf{P}, \mathbf{H u} \mathbf{Z}$, and Lane MD. Monitoring energy balance: metabolites of fatty acid synthesis as hypothalamic sensors. Annual review of biochemistry 74: 515-534, 2005.

13. EImquist JK, Ahima RS, Elias CF, Flier JS, and Saper CB. Leptin activates distinct projections from the dorsomedial and ventromedial hypothalamic nuclei. Proceedings of the National Academy of Sciences of the United States of America 95: 741-746, 1998. 
14. Fang XL, Shu G, Yu JJ, Wang LN, Yang J, Zeng QJ, Cheng X, Zhang ZQ, Wang SB, Gao P, Zhu XT, Xi QY, Zhang YL, and Jiang QY. The Anorexigenic Effect of Serotonin Is Mediated by the Generation of NADPH Oxidase-Dependent ROS. PLoS One 8: e53142, 2013.

15. Gao S, Keung W, Serra D, Wang W, Carrasco P, Casals N, Hegardt FG, Moran TH, and Lopaschuk GD. Malonyl-CoA mediates leptin hypothalamic control of feeding independent of inhibition of CPT-1a. American journal of physiology 301: R209-217, 2011.

16. Gao S, Kinzig KP, Aja S, Scott KA, Keung W, Kelly S, Strynadka K, Chohnan S, Smith WW, Tamashiro KL, Ladenheim EE, Ronnett GV, Tu Y, Birnbaum MJ, Lopaschuk GD, and Moran TH. Leptin activates hypothalamic acetyl-CoA carboxylase to inhibit food intake. Proceedings of the National Academy of Sciences of the United States of America 104: 17358-17363, 2007.

17. Gao S, Zhu G, Gao X, Wu D, Carrasco P, Casals N, Hegardt FG, Moran TH, and Lopaschuk GD. Important roles of brain-specific carnitine palmitoyltransferase and ceramide metabolism in leptin hypothalamic control of feeding. Proceedings of the National Academy of Sciences of the United States of America 108: 9691-9696, 2011.

18. Grill HJ. Distributed neural control of energy balance: contributions from hindbrain and hypothalamus. Obesity 14 Suppl 5: 216S-221S, 2006.

19. Hawke Z, Ivanov TR, Bechtold DA, Dhillon H, Lowell BB, and Luckman SM. PACAP neurons in the hypothalamic ventromedial nucleus are targets of central leptin signaling. J Neurosci 29: 14828-14835, 2009.

20. He W, Lam TK, Obici S, and Rossetti L. Molecular disruption of hypothalamic nutrient sensing induces obesity. Nature neuroscience 9: 227-233, 2006.

21. Jacob RJ, Dziura J, Medwick MB, Leone P, Caprio S, During M, Shulman GI, and Sherwin RS. The effect of leptin is enhanced by microinjection into the ventromedial hypothalamus. Diabetes 46: 150-152, 1997.

22. Jaillard T, Roger M, Galinier A, Guillou P, Benani A, Leloup C, Casteilla L, Penicaud L, and Lorsignol A. Hypothalamic reactive oxygen species are required for insulin-induced food intake inhibition: an NADPH oxidase-dependent mechanism. Diabetes 58: 1544-1549, 2009.

23. Jauregui O, Sierra AY, Carrasco P, Gratacos E, Hegardt FG, and Casals N. A new LC-ESI-MS/MS method to measure long-chain acylcarnitine levels in cultured cells. Analytica chimica acta 599: 1-6, 2007.

24. Kang L, Routh VH, Kuzhikandathil EV, Gaspers LD, and Levin BE. Physiological and molecular characteristics of rat hypothalamic ventromedial nucleus glucosensing neurons. Diabetes 53: 549-559, 2004.

25. Kernie SG, Liebl DJ, and Parada LF. BDNF regulates eating behavior and locomotor activity in mice. Embo $J$ 19: 1290-1300, 2000.

26. Kim KW, Zhao L, Donato J, Jr., Kohno D, Xu Y, Elias CF, Lee C, Parker KL, and Elmquist JK. Steroidogenic factor 1 directs programs regulating diet-induced thermogenesis and leptin action in the ventral medial hypothalamic nucleus. Proceedings of the National Academy of Sciences of the United States of America 108: 10673-10678, 2011.

27. King BM. The rise, fall, and resurrection of the ventromedial hypothalamus in the regulation of feeding behavior and body weight. Physiol Behav 87: 221-244, 2006.

28. Klockener T, Hess S, Belgardt BF, Paeger L, Verhagen LA, Husch A, Sohn JW, Hampel B, Dhillon H, Zigman JM, Lowell BB, Williams KW, Elmquist JK, Horvath TL, Kloppenburg P, and Bruning JC. High-fat feeding promotes obesity via insulin receptor/PI3K-dependent inhibition of SF-1 VMH neurons. Nature neuroscience 14: 911-918, 2011. 
29. Komori T, Morikawa Y, Nanjo K, and Senba E. Induction of brain-derived neurotrophic factor by leptin in the ventromedial hypothalamus. Neuroscience 139: 1107$1115,2006$.

30. Lane MD, Hu Z, Cha SH, Dai Y, Wolfgang M, and Sidhaye A. Role of malonylCoA in the hypothalamic control of food intake and energy expenditure. Biochem Soc Trans 33: 1063-1067, 2005.

31. Loftus TM, Jaworsky DE, Frehywot GL, Townsend CA, Ronnett GV, Lane MD, and Kuhajda FP. Reduced food intake and body weight in mice treated with fatty acid synthase inhibitors. Science (New York, NY 288: 2379-2381, 2000.

32. Lopaschuk GD, Ussher JR, and Jaswal JS. Targeting intermediary metabolism in the hypothalamus as a mechanism to regulate appetite. Pharmacol Rev 62: 237-264, 2010.

33. Lopez M, Lage R, Saha AK, Perez-Tilve D, Vazquez MJ, Varela L, SangiaoAlvarellos S, Tovar S, Raghay K, Rodriguez-Cuenca S, Deoliveira RM, Castaneda T, Datta R, Dong JZ, Culler M, Sleeman MW, Alvarez CV, Gallego R, Lelliott CJ, Carling D, Tschop MH, Dieguez C, and Vidal-Puig A. Hypothalamic fatty acid metabolism mediates the orexigenic action of ghrelin. Cell metabolism 7: 389-399, 2008.

34. Lopez M, Lelliott CJ, Tovar S, Kimber W, Gallego R, Virtue S, Blount M, Vazquez MJ, Finer N, Powles TJ, O'Rahilly S, Saha AK, Dieguez C, and Vidal-Puig AJ. Tamoxifen-induced anorexia is associated with fatty acid synthase inhibition in the ventromedial nucleus of the hypothalamus and accumulation of malonyl-CoA. Diabetes 55: 1327-1336, 2006.

35. Majdic G, Young M, Gomez-Sanchez E, Anderson P, Szczepaniak LS, Dobbins RL, McGarry JD, and Parker KL. Knockout mice lacking steroidogenic factor 1 are a novel genetic model of hypothalamic obesity. Endocrinology 143: 607-614, 2002.

36. McGarry JD, Leatherman GF, and Foster DW. Carnitine palmitoyltransferase I. The site of inhibition of hepatic fatty acid oxidation by malonyl-CoA. The Journal of biological chemistry 253: 4128-4136, 1978.

37. Mera P, Bentebibel A, Lopez-Vinas E, Cordente AG, Gurunathan C, Sebastian D, Vazquez I, Herrero L, Ariza X, Gomez-Puertas P, Asins G, Serra D, Garcia J, and Hegardt FG. C75 is converted to C75-CoA in the hypothalamus, where it inhibits carnitine palmitoyltransferase 1 and decreases food intake and body weight. Biochemical pharmacology 77: 1084-1095, 2009.

38. Morillas M, Gomez-Puertas P, Bentebibel A, Selles E, Casals N, Valencia A, Hegardt FG, Asins G, and Serra D. Identification of conserved amino acid residues in rat liver carnitine palmitoyltransferase I critical for malonyl-CoA inhibition. Mutation of methionine 593 abolishes malonyl-CoA inhibition. The Journal of biological chemistry 278: 9058-9063, 2003.

39. Obici S, Feng Z, Arduini A, Conti R, and Rossetti L. Inhibition of hypothalamic carnitine palmitoyltransferase-1 decreases food intake and glucose production. Nature medicine 9: 756-761, 2003.

40. Obici S, Feng Z, Morgan K, Stein D, Karkanias G, and Rossetti L. Central administration of oleic acid inhibits glucose production and food intake. Diabetes 51: 271275, 2002.

41. Pelleymounter MA, Cullen MJ, and Wellman CL. Characteristics of BDNFinduced weight loss. Exp Neurol 131: 229-238, 1995.

42. Perkins MN, Rothwell NJ, Stock MJ, and Stone TW. Activation of brown adipose tissue thermogenesis by the ventromedial hypothalamus. Nature 289: 401-402, 1981.

43. Price N, van der Leij F, Jackson V, Corstorphine C, Thomson R, Sorensen A, and Zammit $\mathbf{V}$. A novel brain-expressed protein related to carnitine palmitoyltransferase I. Genomics 80: 433-442, 2002. 
44. Riediger T. The receptive function of hypothalamic and brainstem centres to hormonal and nutrient signals affecting energy balance. Proc Nutr Soc 71: 463-477, 2012.

45. Robinson IN and Zammit VA. Sensitivity of carnitine acyltransferase I to malonlyCoA inhibition in isolated rat liver mitochondria is quantitatively related to hepatic malonylCoA concentration in vivo. Biochem J 206: 177-179, 1982.

46. Ross RA, Rossetti L, Lam TK, and Schwartz GJ. Differential effects of hypothalamic long-chain fatty acid infusions on suppression of hepatic glucose production. American journal of physiology Endocrinology and metabolism 299: E633-639, 2010.

47. Schwartz MW, Baskin DG, Kaiyala KJ, and Woods SC. Model for the regulation of energy balance and adiposity by the central nervous system. The American journal of clinical nutrition 69: 584-596, 1999.

48. Schwartz MW, Woods SC, Porte D, Jr., Seeley RJ, and Baskin DG. Central nervous system control of food intake. Nature 404: 661-671, 2000.

49. Schwinkendorf DR, Tsatsos NG, Gosnell BA, and Mashek DG. Effects of central administration of distinct fatty acids on hypothalamic neuropeptide expression and energy metabolism. International journal of obesity 35: 336-344, 2011.

50. Sierra AY, Gratacos E, Carrasco P, Clotet J, Urena J, Serra D, Asins G, Hegardt FG, and Casals N. CPT1c is localized in endoplasmic reticulum of neurons and has carnitine palmitoyltransferase activity. The Journal of biological chemistry 283: 6878-6885, 2008.

51. Stanley S, Wynne K, McGowan B, and Bloom S. Hormonal regulation of food intake. Physiol Rev 85: 1131-1158, 2005.

52. Unger TJ, Calderon GA, Bradley LC, Sena-Esteves M, and Rios M. Selective deletion of Bdnf in the ventromedial and dorsomedial hypothalamus of adult mice results in hyperphagic behavior and obesity. The Journal of neuroscience : the official journal of the Society for Neuroscience 27: 14265-14274, 2007.

53. Vance D, Goldberg I, Mitsuhashi O, and Bloch K. Inhibition of fatty acid synthetases by the antibiotic cerulenin. Biochemical and biophysical research communications 48: 649-656, 1972.

54. Wade GN and Zucker I. Modulation of food intake and locomotor activity in female rats by diencephalic hormone implants. J Comp Physiol Psychol 72: 328-336, 1970.

55. Wang C, Bomberg E, Billington CJ, Levine AS, and Kotz CM. Brain-derived neurotrophic factor (BDNF) in the hypothalamic ventromedial nucleus increases energy expenditure. Brain research 1336: 66-77, 2010.

56. Wang C, Bomberg E, Levine A, Billington C, and Kotz CM. Brain-derived neurotrophic factor in the ventromedial nucleus of the hypothalamus reduces energy intake. American journal of physiology Regulatory, integrative and comparative physiology 293: R1037-1045, 2007.

57. Winder WW, Arogyasami J, Elayan IM, and Cartmill D. Time course of exerciseinduced decline in malonyl-CoA in different muscle types. The American journal of physiology 259: E266-271, 1990.

58. Wolfgang MJ, Kurama T, Dai Y, Suwa A, Asaumi M, Matsumoto S, Cha SH, Shimokawa T, and Lane MD. The brain-specific carnitine palmitoyltransferase-1c regulates energy homeostasis. Proceedings of the National Academy of Sciences of the United States of America 103: 7282-7287, 2006.

59. Xu B, Goulding EH, Zang K, Cepoi D, Cone RD, Jones KR, Tecott LH, and Reichardt LF. Brain-derived neurotrophic factor regulates energy balance downstream of melanocortin-4 receptor. Nature neuroscience 6: 736-742, 2003.

60. Yeo GS, Connie Hung CC, Rochford J, Keogh J, Gray J, Sivaramakrishnan S, O'Rahilly S, and Farooqi IS. A de novo mutation affecting human TrkB associated with severe obesity and developmental delay. Nature neuroscience 7: 1187-1189, 2004. 


\section{Figure Legend}

Fig. 1 Administration of the CPT-1a adenoviruses induces overexpressions of the CPT-1a protein: The adenovirus without the CPT-1a insert (null), encoding wild-type CPT-1a (CPT-1a wt), or M593S mutant CPT-1a (CPT-1a mt) was administered into the hypothalamic ventromedial nucleus (VMN). One week after the adenovirus administration, the rats were euthanized. The hypothalamic nuclei were dissected, and the CPT-1a and CPT-1c protein levels were measured by Western blot analysis. Three to four $(n=3-4)$ representative blots from the dissected VMN area of each group are shown. The ratio of the band intensity of CPT-1a or CPT-1c to that of tubulin was quantitated. *, ** wt or mt vs. null, $\mathrm{P}<0.05$.

Fig. 2 Administration of the CPT-1a adenoviruses induces overexpressions of the CPT-1a protein: One week following VMN administration of the adenoviruses (null, CPT-1a wt and CPT1a $\mathrm{mt} ; \mathrm{n}=8-9$ ), the rats were euthanized. The CPT-1 acyltransferase activities in individual hypothalamic nuclei (A) and the long-chain acylcarnitine levels in VMN and Arc (B) were measured. *** wt or mt vs. null, $\mathrm{P}<0.05$.

Fig. 3 VMN administration of the CPT-1a adenoviruses induces orexigenic effects: Following VMN administration of the adenoviruses (null, CPT-1a wt and CPT-1a mt; $\mathrm{n}=4-6$ ), daily overnight food intake (A) and body weight (B) were monitored. The food intake of the day before viral injection (day 0) was used as the basal level. In the rats with VMN administration of CPT-1a viruses, the values from the rats with significant increases of CPT-1a activity as compared to the Arc (by acylcarnitine levels) were included in the data analysis. Food intake (A): from day 5 through 8 , * wt or mt vs. null, $\mathrm{P}<0.05$; day 8 , ** mt vs. wt, $\mathrm{P}<0.05$. Body weight (B): from day 4 through 8 , * wt or mt vs. null, $\mathrm{P}<0.05$. (C) After 8 days following VMN administration of the adenoviruses ( $\mathrm{n}=6-10)$, long-chain fatty acyl-CoA's levels in the VMN were measured. *, ** wt or mt vs. null, $\mathrm{P}<0.05$. 
Fig. 4 VMN overexpression of the mutant CPT-1a blocks etomoxir-induced changes in feeding and body weight: The null, CPT-1a wt, and CPT-1a mt adenoviruses were administered into the

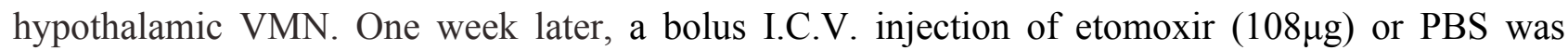
given to the rats. (A and B) The VMN levels of long-chain acylcarnitines (A, n=7-9) and long-chain fatty acyl-CoA's $(B, n=6-10)$ at $8 \mathrm{~h}$ after the injection were measured. Percent decreases of acylcarnitine levels and increases of acyl-CoA levels by etomoxir (as compared with PBS) are shown. * wt or mt vs. null, $\mathrm{P}<0.05$. (C and D) Overnight food intake (C) and body weight (D) following the injection were monitored $(n=4-5)$. Food intake: Percent decreases by etomoxir (as compared with PBS) are shown; * wt or mt vs. null, $\mathrm{P}<0.05$. Body weight: etomoxir treatment significantly reduces food intake across the three groups (null, CPT-1a wt and CPT-1a mt); * etomoxir/mt vs. etomoxir/wt or etomoxir/null, $\mathrm{P}<0.05$.

Fig. 5 Arc overexpression of the mutant CPT-1a does not affect etomoxir-induced changes in feeding and body weight: The null, CPT-1a wt, and CPT-1a mt adenoviruses were administered into the hypothalamic Arc. One week later, a bolus I.C.V. injection of etomoxir $(108 \mu \mathrm{g})$ or PBS was given to the rats. (A and B) The Arc levels of long-chain acylcarnitines $(A, n=8-12)$ and longchain fatty acyl-CoA's $(B, n=6-11)$ at $8 \mathrm{~h}$ after the injection were measured. Percent decreases of acylcarnitine levels and increases of acyl-CoA levels by etomoxir (as compared with PBS) are shown. Long-chain acylcarnitines: * wt or mt vs. null, $\mathrm{P}<0.05$; long-chain acyl-CoA's: * mt vs. wt, $\mathrm{P}<0.05$. (C and D) Overnight food intake (C) and body weight (D) following the injection were monitored $(n=6)$. Food intake: Percent decreases by etomoxir (as compared with PBS) are shown; body weight: etomoxir treatment significantly reduces food intake across the three groups (null, CPT-1a wt and CPT-1a mt).

Fig. 6 VMN overexpression of the mutant CPT-1a blocks cerulenin-induced changes in feeding and body weight: One week following VMN administration of the null, CPT-1a wt, and 
CPT-1a mt adenoviruses, a bolus I.C.V. injection of cerulenin (125 $\mu \mathrm{g}$, in 25\% DMSO) or 25\% DMSO (vehicle) was given. Overnight food intake $(\mathrm{A})$ and body weight $(\mathrm{B})$ were monitored $(\mathrm{n}=$ 4). Food intake: Percent decreases by cerulenin (as compared with the vehicle) are shown; * wt or mt vs. null, $\mathrm{P}<0.05$. Body weight: cerulenin treatment significantly reduces food intake across the three groups (null, CPT-1a wt and CPT-1a mt); * cerulenin/mt vs. cerulenin/wt or cerulenin/null, $\mathrm{P}<0.05$.

Fig. 7 Fasting and refeeding alter VMN levels of malonyl-CoA, long-chain acylcarnitines and long-chain acyl-CoA's: The rats were fasted for $24 \mathrm{~h}(\mathrm{~F} 24 \mathrm{~h})$ or $48 \mathrm{~h}$ (F48h). At the end of the 48hfasting, some rats were refed for $3 h(R F 3 h)$ or $24 h(R F 24 h)$. Ad libitum fed (fed) was used as the control. VMN levels of malonyl-CoA (A, n=6-9), long-chain acylcarnitines $(B, n=2-5)$ and longchain acyl-CoA's $(\mathrm{C}, \mathrm{n}=6-9)$ were measured. Malonyl-CoA and long-chain acylcarnitines: *, ** F24h or F48h vs. fed, P < 0.05; \#,\#\# RF3h or RF24h vs. F48h, P < 0.05. Long-chain acyl-CoA's: *F48h vs. F24h or fed, $\mathrm{P}<0.05$; \#,\#\# RF3h or RF24h vs. F48h, $\mathrm{P}<0.05$. 
protein levels of CPT-1 isoforms in VMN



CPT-1a

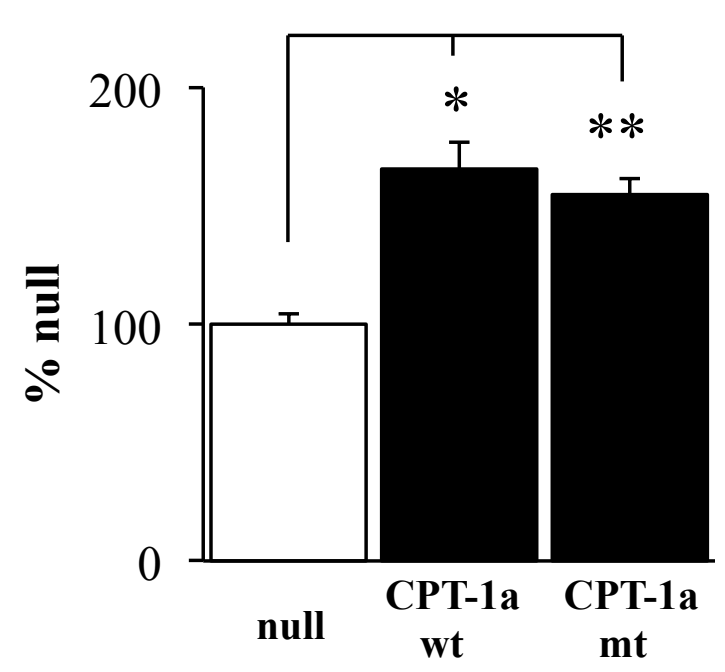

CPT-1c



Fig. 1 
(A) CPT-1 activities in hypothalamic nuclei

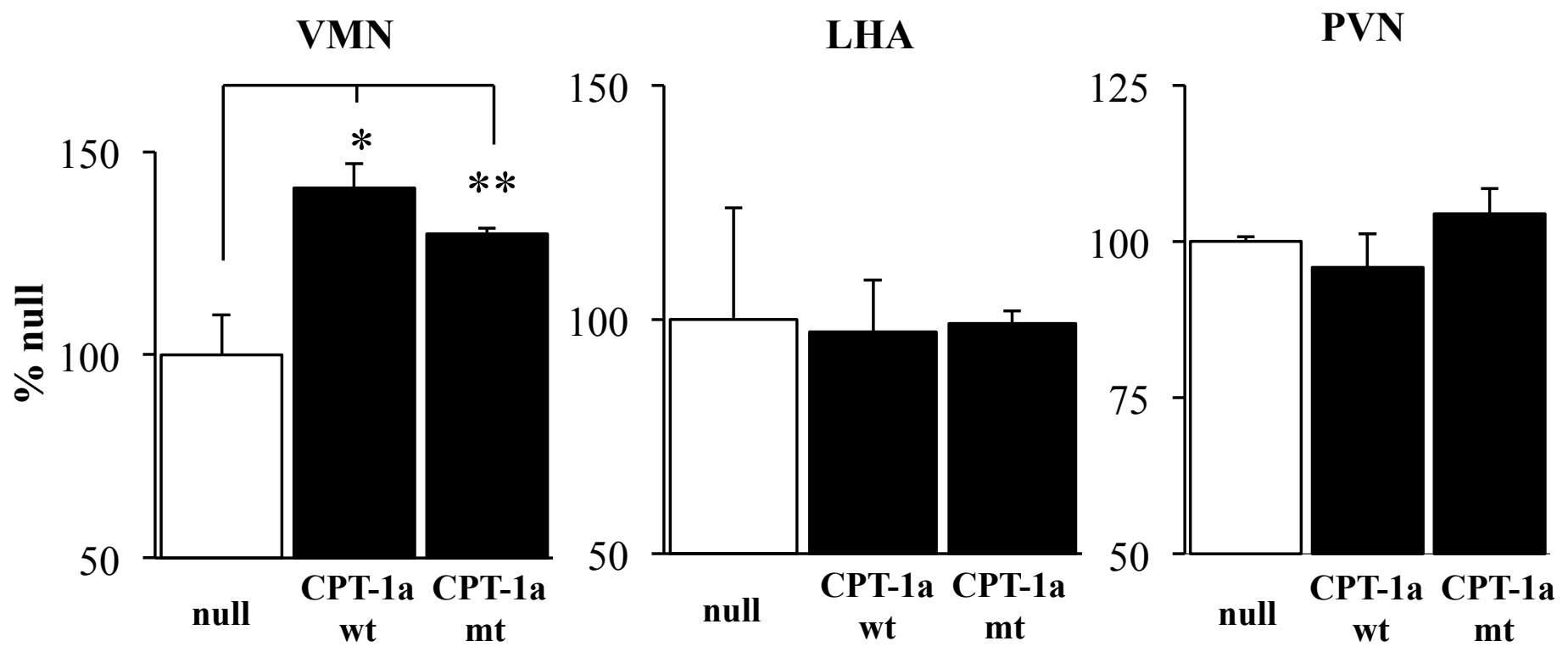

(B) long-chain acylcarnitine levels in VMN and Arc
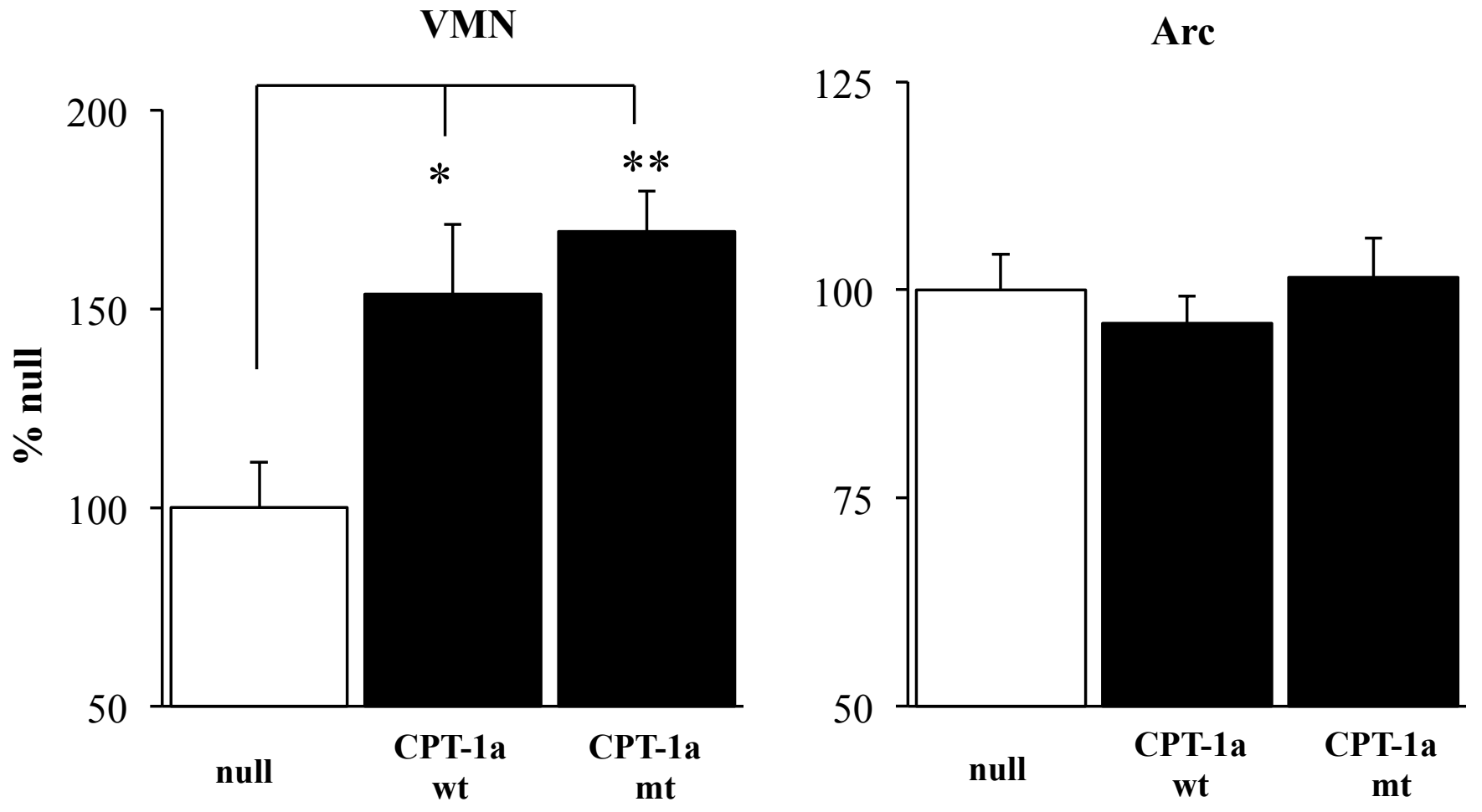

Fig. 2 
(A) daily food intake

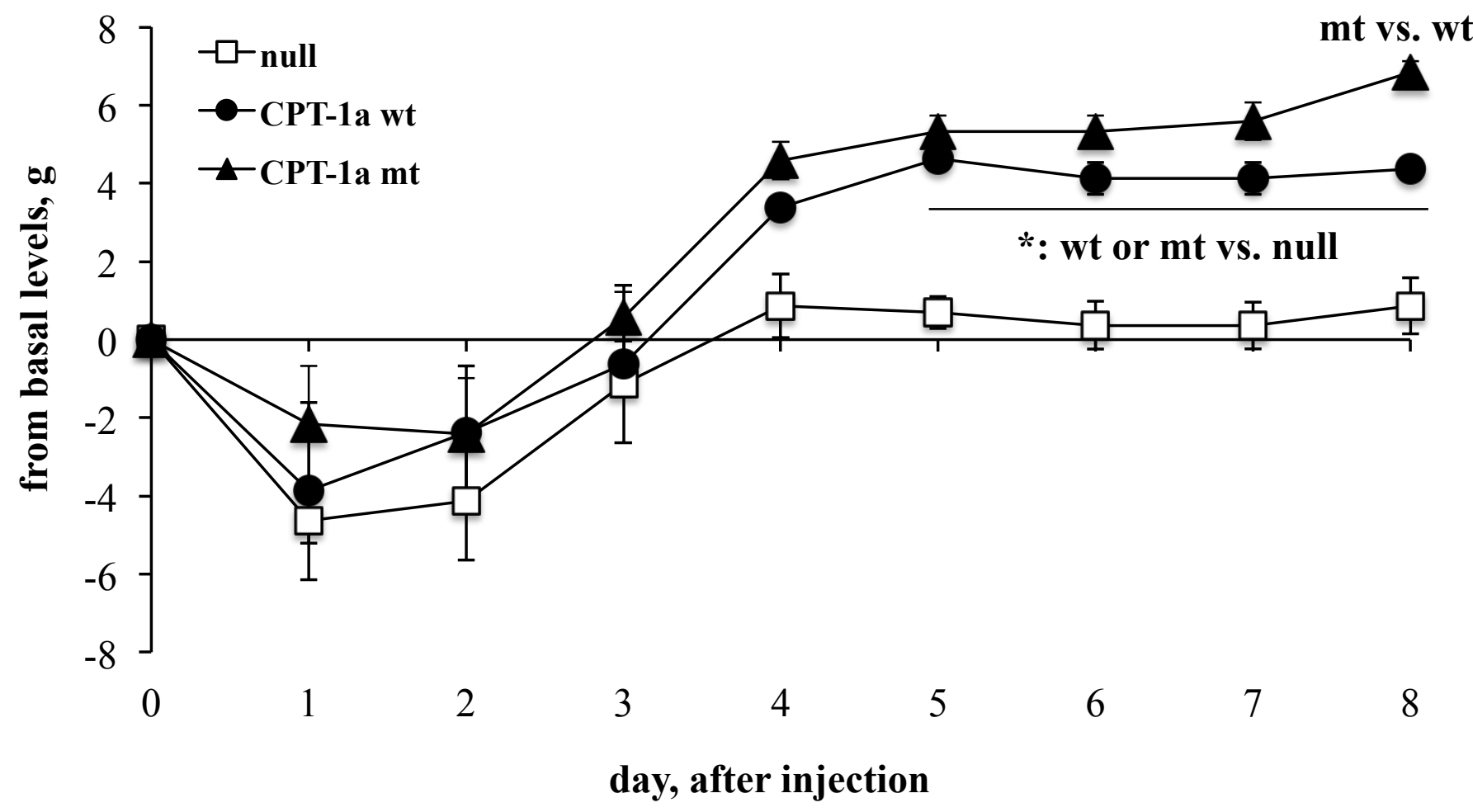

(B) daily body weight

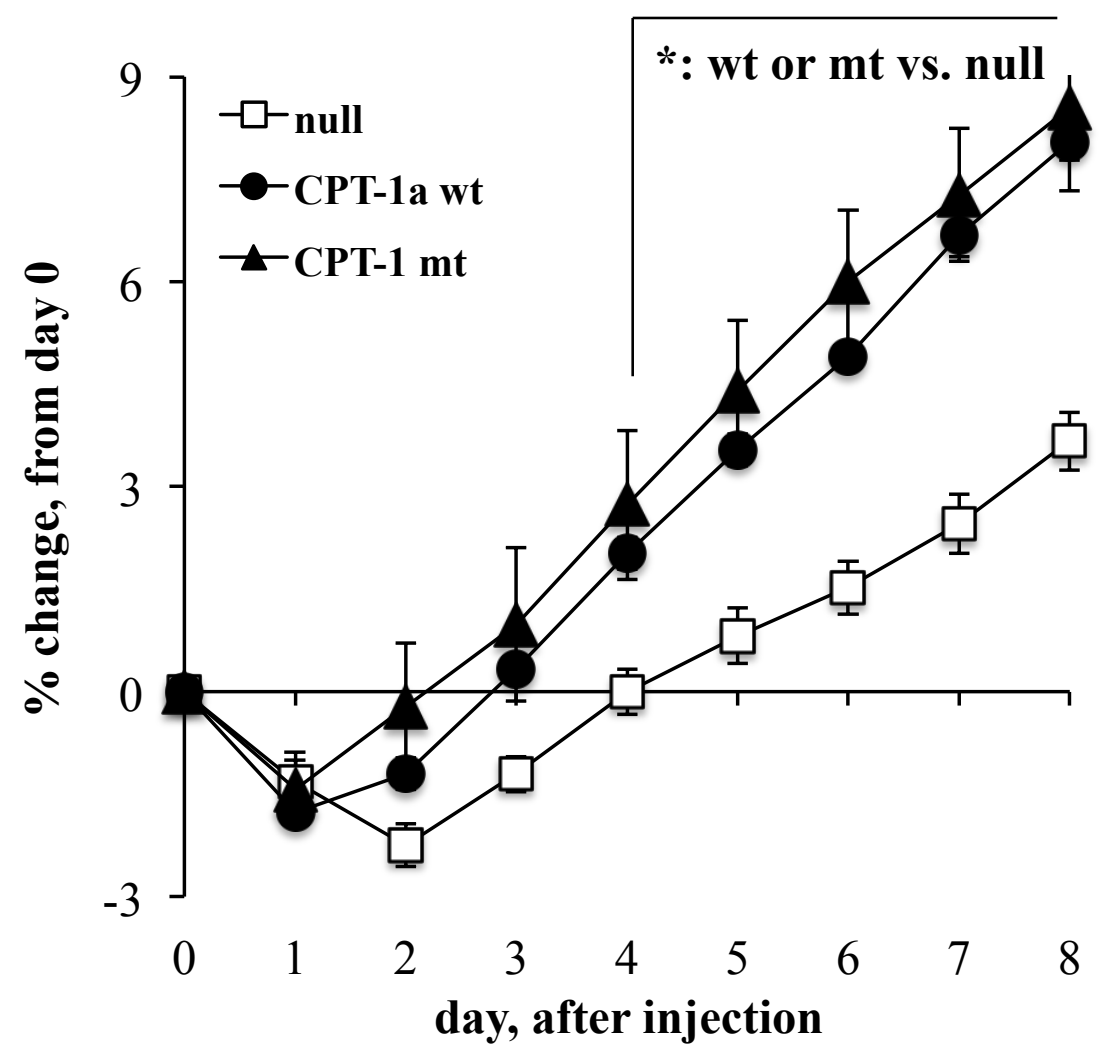

(C) long-chain acyl-CoA levels



Fig. 3 
(A) long-chain acylcarnitines

(B) long-chain acyl-CoA's
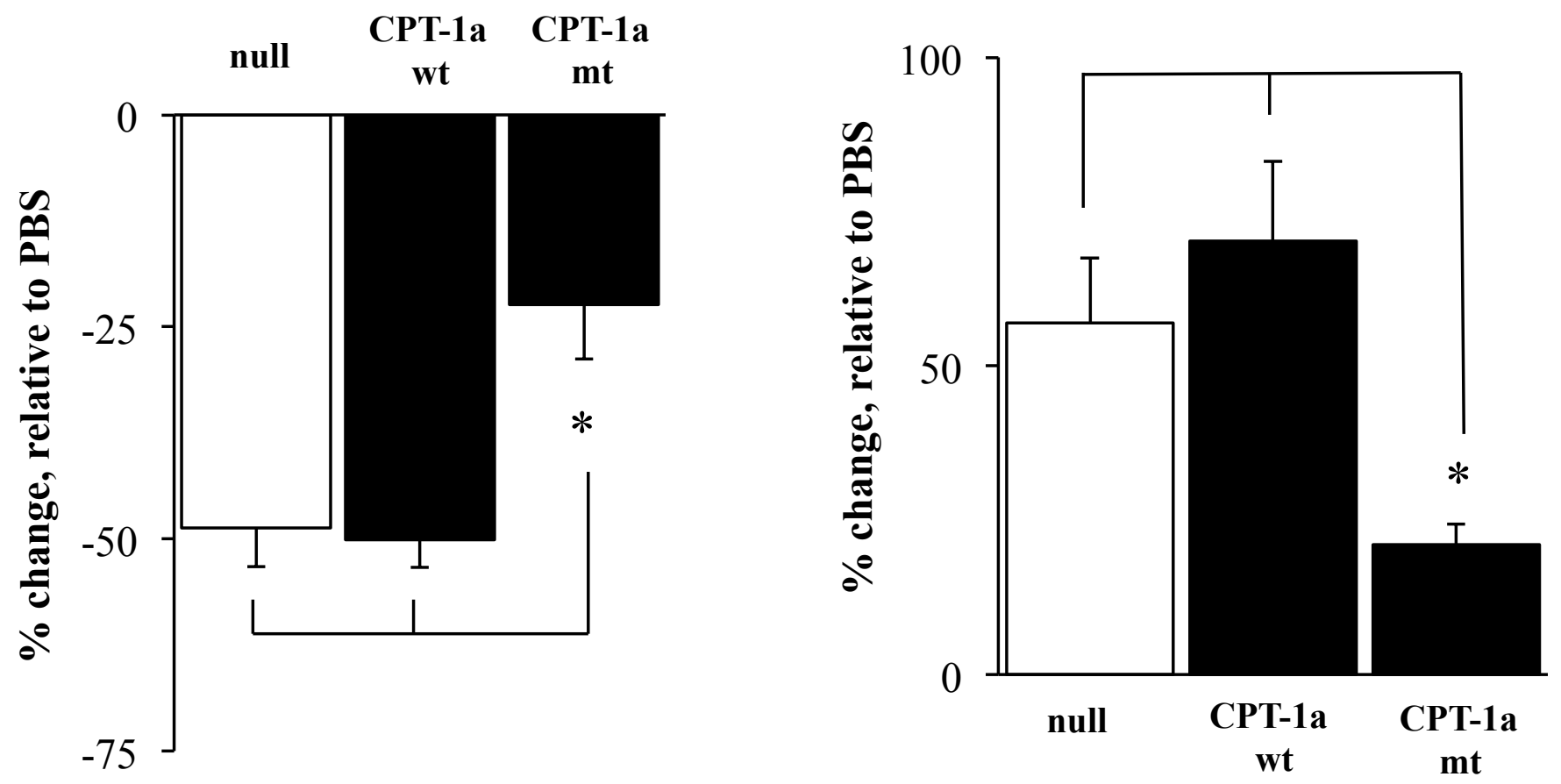

(C) food intake

(D) body weight


Fig. 4 
(A) long-chain acylcarnitines

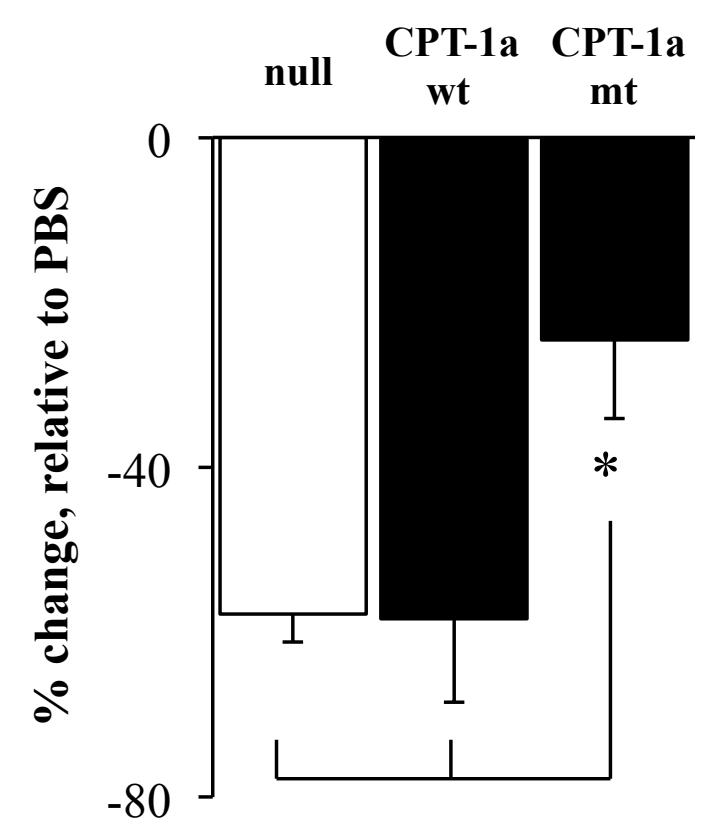

(C) food intake

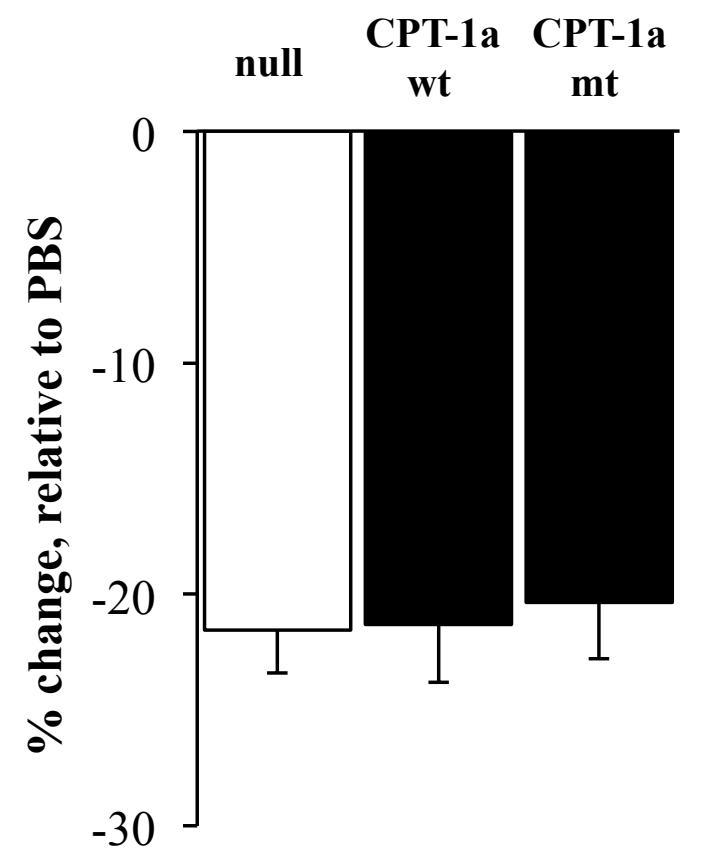

(B) long-chain acyl-CoA's

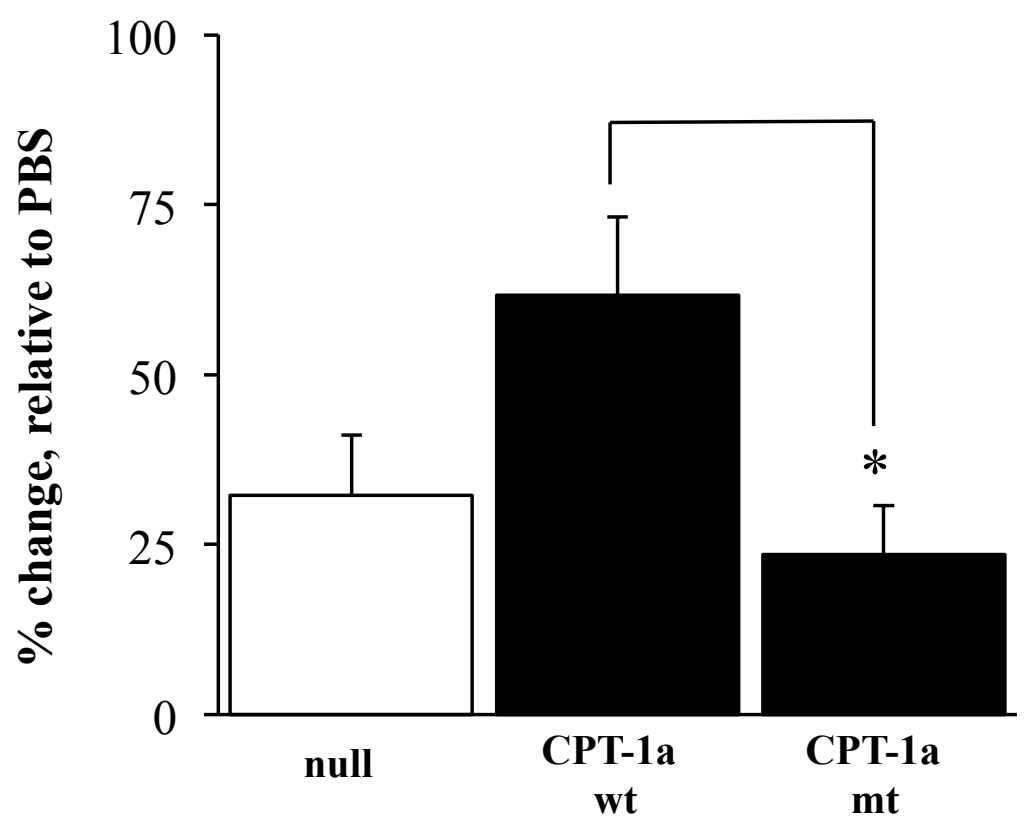

(D) body weight

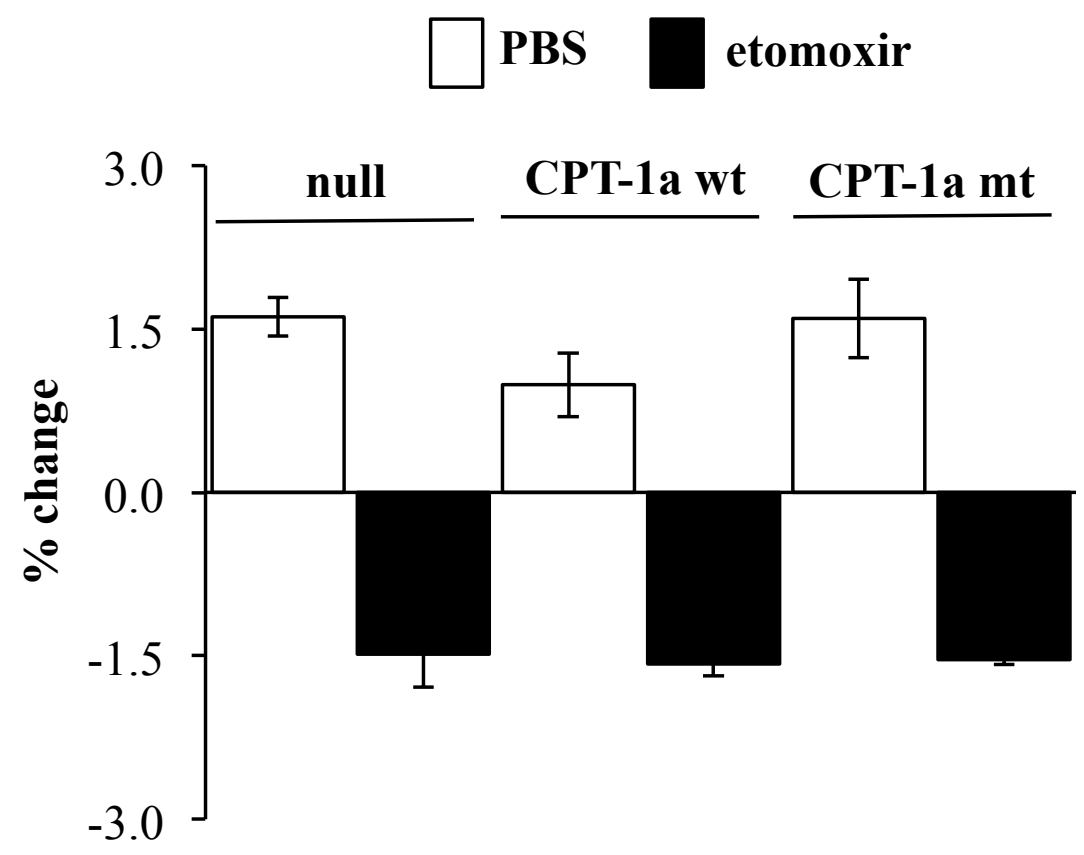

Fig. 5 
(A) food intakes

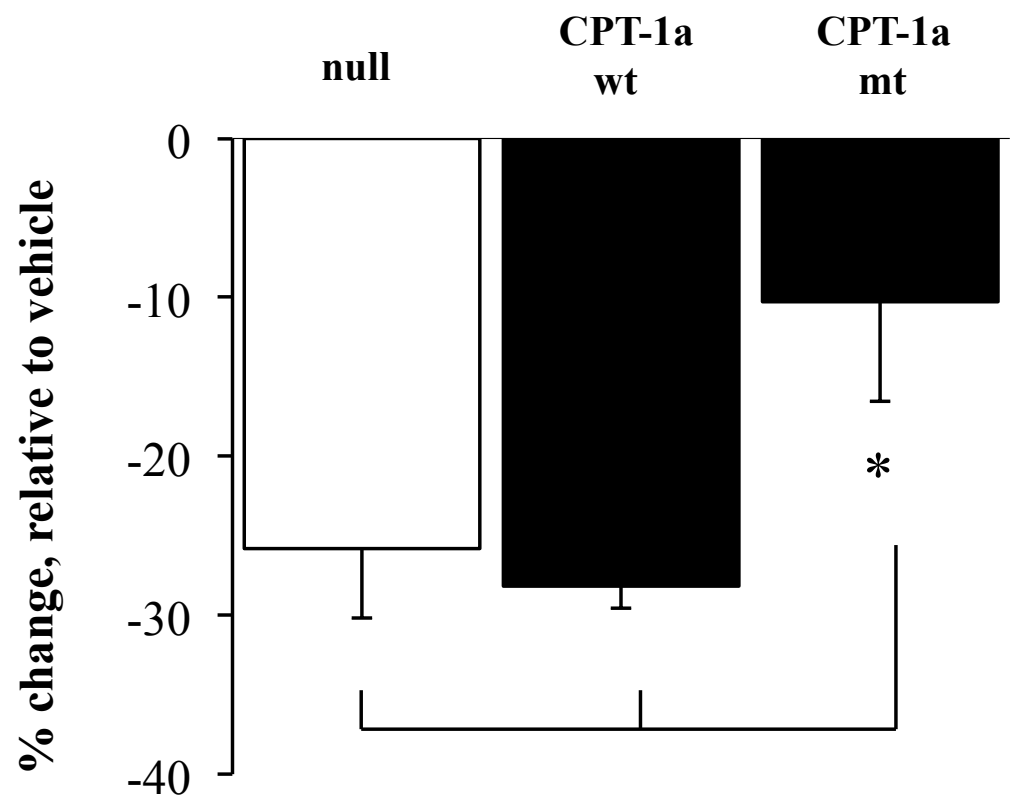

(B) body weight



Fig. 6 
(A) malonyl-CoA

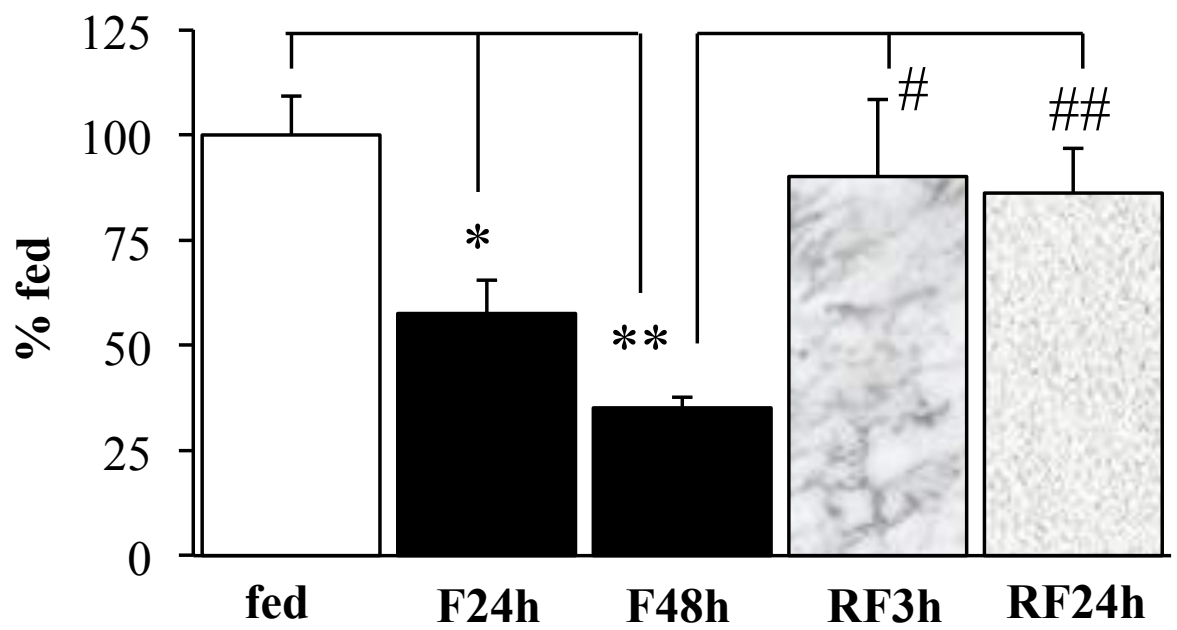

(B) long-chain acylcarnitines

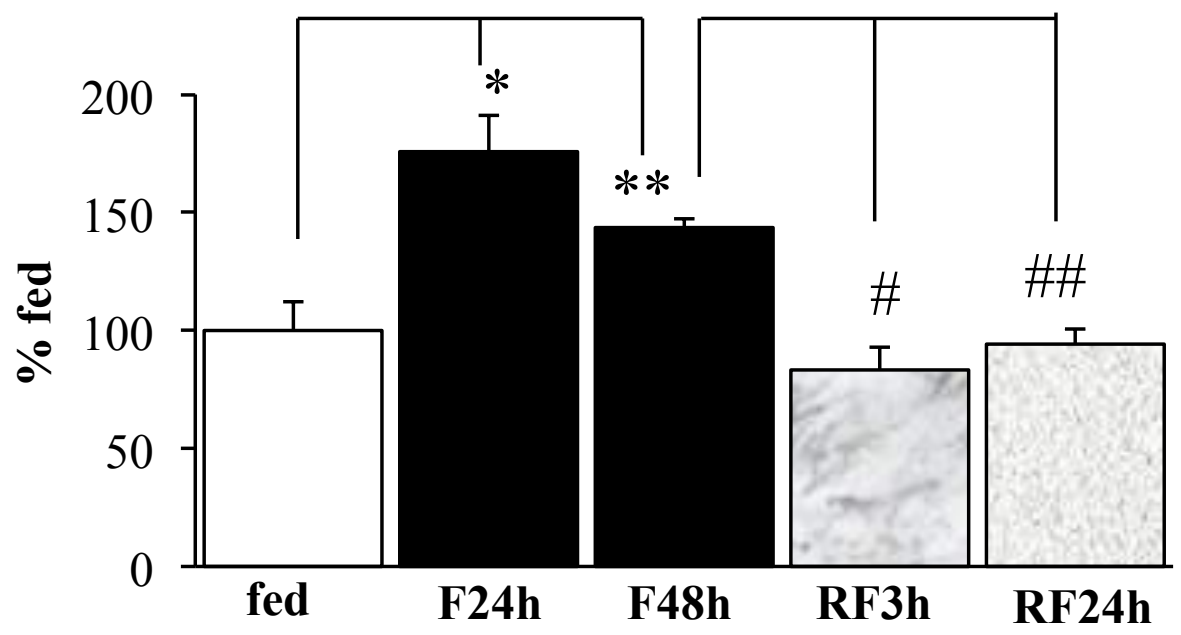

(C) long-chain acyl-CoA's

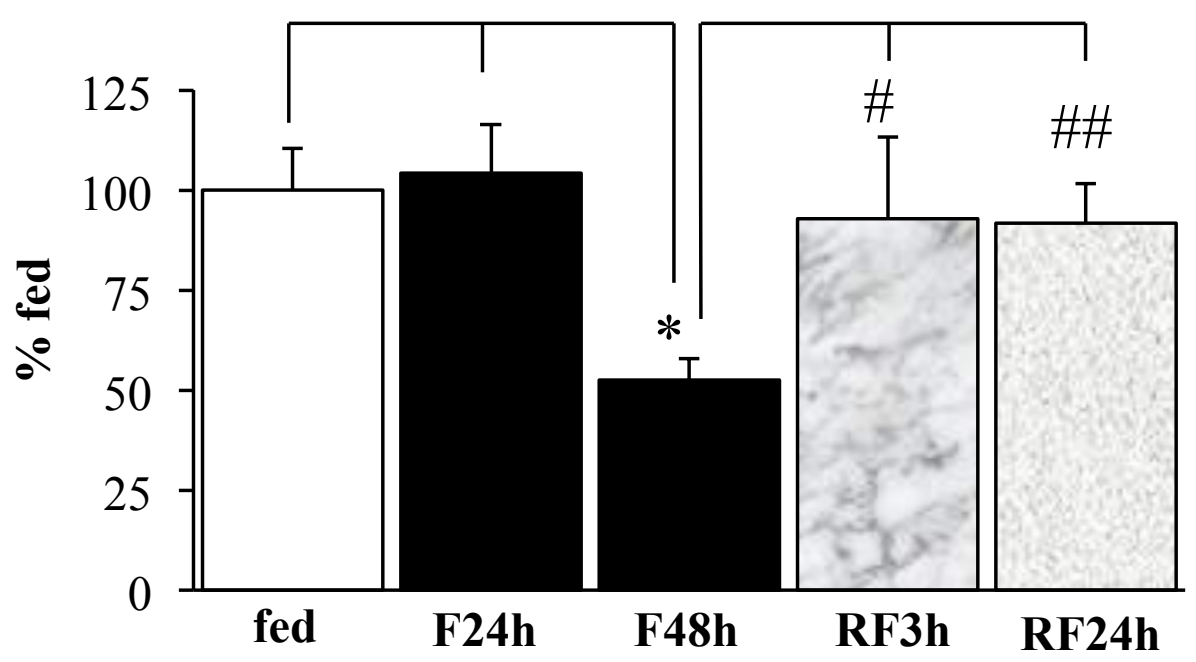

